\title{
The Stages of Drug Discovery and Development Process
}

\section{Amol B. Deore*, Jayprabha R. Dhumane, Hrushikesh V Wagh, Rushikesh B. Sonawane}

\author{
MVP's Institute of Pharmaceutical Sciences, Adgaon, Nashik-422003 (India)
}

\begin{abstract}
A B S T R A C T
Drug discovery is a process which aims at identifying a compound therapeutically useful in curing and treating disease. This process involves the identification of candidates, synthesis, characterization, validation, optimization, screening and assays for therapeutic efficacy. Once a compound has shown its significance in these investigations, it will initiate the process of drug development earlier to clinical trials. New drug development process must continue through several stages in order to make a medicine that is safe, effective, and has approved all regulatory requirements. One overall theme of our article is that the process is sufficiently long, complex, and expensive so that many biological targets must be considered for every new medicine ultimately approved for clinical use and new research tools may be needed to investigate each new target. From initial discovery to a marketable medicine is a long, challenging task. It takes about 12 - 15 years from discovery to the approved medicine and requires an investment of about US $\$ 1$ billion. On an average, a million molecules screened but only a single is explored in late stage clinical trials and is finally made obtainable for patients. This article provides a brief outline of the processes of new drug discovery and development.
\end{abstract}

Key words: Lead optimization, clinical trials, target validation, identification, new drug.

A R T I C L E I N F 0: Received 18 Sep. 2019; $\quad$ Review Completed 25 Nov. 2019; $\quad$ Accepted 14 Dec. 2019; $\quad$ Available online 15 Dec. 2019

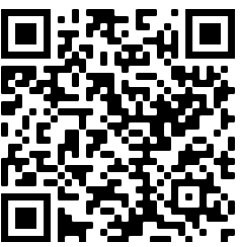

Cite this article as:

Deore, AB, Dhumane JR, Wagh HV, Sonawane RB, The Stages of Drug Discovery and Development Process. Asian Journal of

Pharmaceutical Research and Development. 2019; 7(6):62-67, DOI: http://dx.doi.org/10.22270/ajprd.v7i6.616

*Address for Correspondence:

Amol B. Deore, MVP’s Institute of Pharmaceutical Sciences, Adgaon, Nashik-India

\section{INTRODUCTION}

$\mathrm{D}$ rug discovery is a multifaceted process, which involves identification of a drug chemical therapeutically useful in treating and management of a disease condition. Typically, researchers find out new drugs through new visions into a disease process that permit investigator to design a medicine to stopover or contrary the effects of the disease. ${ }^{[1]}$ The process of drug discovery includes the identification of drug candidates, synthesis, characterization, screening, and assays for therapeutic efficacy. When a molecule avails its satisfactory results in these investigations, it will commence the process of drug development subsequent to clinical trials. Drug discovery and development is an expensive process due to the high budgets of $R \& D$ and clinical trials. It takes almost 12-15 years to develop a single new drug molecule from the time it is discovered when it is available in market for treating patients. ${ }^{[2]}$ The average cost for research and development for each efficacious drug is likely to be $\$ 900$ million to $\$ 2$ billion. This figure includes the cost of the thousands of failures: For every 5,000-10,000 compounds that enter the investigation and development pipeline, ultimately only one attains approval. These statistics challenge imagination, but a brief understanding of the $\mathrm{R} \& \mathrm{D}$ process can explain why so many compounds don't make it and why it takes such a large, lengthy effort to get one medicine to patients. ${ }^{[3]}$ The Success requires immense resources the best scientific and logical minds, highly sophisticated laboratory and technology; and multifaceted project management. It also takes persistence and good fortune. ${ }^{[4]}$ Eventually, the process of drug discovery brings hope, faith and relief to billions of patients. ${ }^{[5]}$ 
Stages of drug discovery and development include:

- Target identification

- Target validation

- lead identification

- lead optimization

- Product characterization
- Formulation and development

- Preclinical research

- Investigational New Drug

- Clinical trials

- New Drug Application

- Approval

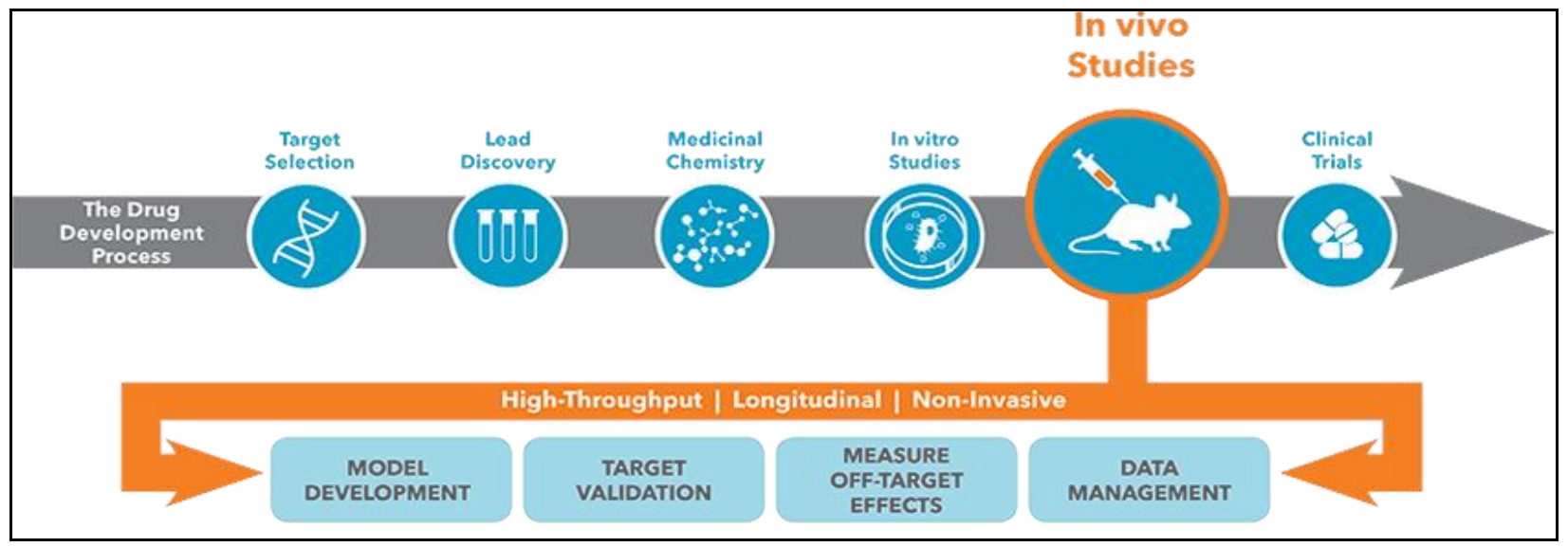

Figure 1: Stages of drug discovery and development process

\section{Target Identification}

The first step in the discovery of a drug is identification of the biological origin of a disease, and the potential targets for intervention. Target identification starts with isolating the function of a possible therapeutic target (gene/nucleic acid/protein) and its role in the disease. ${ }^{[6]}$ Identification of the target is followed by characterization of the molecular mechanisms addressed by the target. An ideal target should be efficacious, safe, meet clinical and commercial requirements and be 'druggable'. The techniques used for target identification may be based on principles of molecular biology, biochemistry, genetics, biophysics, or other disciplines. ${ }^{[7]}$

Approaches:

- Data mining using bioinformatics

- identifying, selecting and prioritizing potential disease targets

- Genetic association

- genetic polymorphism and connection with the disease

- Expression profile - changes in mRNA/protein levels

- Pathway and phenotypic analysis - In vitro cell-based mechanistic studies

- Functional screening - knockdown, knockout or using target specific tools $^{[8]}$

\section{Target Validation}

Target validation is the process by which the expected molecular target - for example gene, protein or nucleic acid of a small molecule is certified. Target validation includes: determining the structure activity relationship (SAR) of analogs of the small molecule; generating a drug-resistant mutant of the presumed target; knockdown or over expression of the presumed target; and monitoring the known signaling systems downstream of the presumed target. ${ }^{[9]}$

Target validation is the process of demonstrating the functional role of the identified target in the disease phenotype. Whilst the validation of a drug's efficacy and toxicity in numerous disease-relevant cell models and animal models is extremely valuable - the ultimate test is whether the drug works in a clinical setting. ${ }^{[10]}$

Target validation can be broken down in to two key steps.

Reproducibility: Once a drug target is identified, whether it be via a specific technique or from review of literature, the first step is to repeat the experiment to confirm that it can be successfully reproduced. The target validation technique includes affinity chromatography, expression-cloning, protein microarray, reverse transfected cell microarray, biochemical suppression, siRNA, DNA microarray, system biology and study of existing drugs. ${ }^{[11,12]}$

Introduce variation to the ligand (drug)-targetenvironment

- Genetic manipulation of target genes (in vitro) knocking down the gene (shRNA, siRNA, miRNA), knocking out the gene (CRISPR), knocking in the genes (viral transfection of mutant genes)

\section{- Antibodies} interacting to the target with high affinity and blocking further interactions

- Chemical genomics chemical approaches against genome encoding protein ${ }^{[13]}$

\section{Identification of Lead}

A chemical lead is defined as a synthetically stable, feasible, and drug like molecule active in primary and secondary assays with acceptable specificity, affinity and selectivity for 
the target receptor. This requires definition of the structure activity relationship as well as determination of synthetic feasibility and preliminary evidence of in vivo efficacy and target engagement. Characteristics of a chemical lead are:

- SAR defined

- Drug ability (preliminary toxicity, hERG)

- Synthetic feasibility

- Select mechanistic assays

- In vitro assessment of drug resistance and efflux potential

- Evidence of in vivo efficacy of chemical class

- PK/Toxicity of chemical class known based on preliminary toxicity or in silico studies

In order to decrease the number of compounds that fail in the drug development process, a drug ability assessment is often conducted. This assessment is important in transforming a compound from a lead molecule into a drug. For a compound to be considered druggable it should have the potential to bind to a specific target; however, also important is the compound's pharmacokinetic profile regarding absorption, distribution, metabolism, and excretion. Other assays will evaluate the potential toxicity of the compound in screens such as the Ames test and cytotoxicity assay. ${ }^{[14]}$

\section{Lead Optimization}

Lead optimization is the process by which a drug candidate is designed after an initial lead compound is identified. The process involves iterative series of synthesis and characterization of a potential drug to build up a representation of in what way chemical structure and activity are related in terms of interactions with its targets and its metabolism.

In initial drug discovery, the resulting leads from hit-to-lead high throughput screening tests undergo lead optimization, to identify promising compounds. Potential leads are evaluated for a range of properties, including selectivity and binding mechanisms during lead optimization, as the final step in early stage drug discovery. The purpose of lead optimization is to maintain favorable properties in lead compounds, while improving on deficiencies in lead structure. In order to produce a pre-clinical drug candidate, the chemical structures of lead compounds (small molecules or biologics) need to be altered to improve target specificity and selectivity. Pharmacodynamic and pharmacokinetic parameters and toxicological properties are also evaluated. Labs must acquire data on the toxicity, efficacy, stability and bioavailability of leads, in order to accurately characterize the compound and establish the route of optimization. ${ }^{[15]}$

Researchers in drug discovery need rapid methods to narrow down the selection of drug candidates for this downstream selectivity profiling and further investigation. High throughput DMPK (drug metabolism and pharmacokinetics) screens have become an essential part of lead optimization, facilitating the understanding and prediction of in vivo pharmacokinetics using in vitro tests. In order to make new drugs with higher potency and safety profiles, chemical modifications to the structure of candidate drugs are made through optimization.
Automated screening systems are becoming an important part of pharmaceutical and biopharmaceutical drug discovery labs. Mass spectrometry is used for the detection and quantitation of metabolites. MALDI imaging is a key technique for evaluating drug candidates and their metabolites in tissue structure rapidly and accurately. Additionally, NMR Fragment-based Screening (FBS) in the pharmaceutical industry has become a widely applied method for the discovery and optimization of lead molecules in targeted screening campaigns. ${ }^{[16]}$

\section{Product Characterization}

When any new drug molecule shows a promising therapeutic activity, then the molecule is characterized by its size, shape, strength, weakness, use, toxicity, and biological activity. Early stages of pharmacological studies are helpful to characterize the mechanism of action of the compound.

\section{Formulation and Development}

Pharmaceutical formulation is a stage of drug development during which the physicochemical properties of active pharmaceutical ingredients (APIs) are characterized to produce a bioavailable, stable and optimal dosage form for a specific administration route.

During preformulation studies the following parameters are evaluated:

- Solubility in different media and solvents

- Dissolution of the active pharmaceutical ingredient (API)

- Accelerated Stability Services under various conditions

- Solid state properties (polymorphs, particle size, particle shape etc.)

- Formulation services and capabilities

- Formulation development of new chemical entities (NCE)

- Optimization of existing formulations

- Process development for selected dosage forms

- Novel formulations for improved delivery of existing dosage forms

- Controlled release and sustained release formulations

- Self-emulsifying drug delivery systems

- Colloidal drug delivery systems

- Sub-micron and nano-emulsions

\section{Preclinical Testing}

Pre-clinical research in drug development process involves evaluation of drug's safety and efficacy in animal species that conclude to prospective human outcome. The preclinical trials also have to acquire approval by corresponding regulatory authorities. The regulatory authorities must ensure that trials are conducted in safe and ethical way and would give approval for only those drugs which are confirm to be safe and effective. ICH has established a basic guideline for technical necessities of acceptable preclinical drug development. ${ }^{[17]}$

The pre-clinical trials can be conducted in two ways: General pharmacology and Toxicology. Pharmacology deals with the pharmacokinetic and pharmacodynamic parameters of drug. It is essential to explore unwanted pharmacological effects in suitable animal models and monitoring them in toxicological studies. Pharmacokinetic studies are very important to make known the safety and efficacy parameters in terms of 
absorption, distribution, metabolism and excretion. These studies give information on absorption rate for diverse routes of administration, which helps in selection of dosage form, distribution, rate of metabolism and elimination; which governs the half-life of the drug. Half-life of the drug clarifies the safety outline of the drug which is the obligatory for a drug to get approved by regulatory agencies. The drug distribution mechanism elucidates the therapeutic effectiveness of the drug as it depends on the drugs bioavailability and its affinity. Drug metabolism provides the probability of through phases of biotransformation process and formation of drug metabolites. It also helps in understanding the reactions as well as enzymes involved in biotransformation. ${ }^{[18]}$

Toxicological studies of the drug can be performed by invitro and in-vivo test which evaluate the toxicological effects of the drug. In-vitro studies can be performed to inspect the direct effects on cell proliferation and phenotype. In-vivo studies can be performed for qualitative and quantitative determination of toxicological effects. As many drugs are species specific, it is essential to select appropriate animal species for toxicity study. In-vivo studies to evaluate pharmacological and toxicological actions, including mode of action, are often used to support the basis of the proposed use of the product in clinical studies. ${ }^{[19]}$

\section{The Investigational New Drug Process (IND)}

Drug developers must file an Investigational New Drug application to FDA before commencement clinical research. ${ }^{[20]}$ In the IND application, developers must include:

- Preclinical and toxicity study data

- Drug manufacturing information

- Clinical research protocols for studies to be conducted

- Previous clinical research data (if any)

- Information about the investigator/ developer ${ }^{[21]}$

\section{Clinical Research}

Clinical trials are conducted in people (volunteer)and intended to answer specific questions about the safety and efficacy of drugs, vaccines, other therapies, or new methods of using current treatments. Clinical trials follow a specific study protocol that is designed by the researcher or investigator or manufacturer. As the developers design the clinical study, they will consider what they want to complete for each of the different Clinical Research Phases and starts the Investigational New Drug Process (IND), a process they must go through before clinical research begins. Before a clinical trial begins, researchers review prior information about the drug to develop research questions and objectives. ${ }^{[22]}$ Then, they decide:

- Selection criteria for participants

- Number of people take part of the study

- Duration of study

- Dose and route of administration of dosage form

- Assessment of parameters

- Data collection and analysis

\section{Phase 0 clinical trial}

Phase 0 implicates investigative, first-in-human (FIH) trials that are conducted according to FDA guidelines. Phase 0 trials besides termed as human micro dose studies, they have single sub-therapeutic doses given to 10 to 15 volunteers and give pharmacokinetic data or help with imaging specific targets without exerting pharmacological actions. Pharmaceutical industries perform Phase 0 studies to pick which of their drug applicants has the preeminent pharmacokinetic parameters in humans. ${ }^{[24]}$

\section{Phase 1: Safety and dosage}

Phase I trials are the first tests of a drug with a lesser number of healthy human volunteers. In most cases, 20 to 80 healthy volunteers with the disease/condition participate in Phase 1. Patients are generally only used if the mechanism of action of a drug indicates that it will not be tolerated in healthy people. However, if a new drug is proposed for use in diabetes patients, researchers conduct Phase 1 trials in patients with that type of diabetes. Phase 1 studies are closely monitored and collect information about Pharmacodynemics in the human body. Researchers adjust dosage regimen based on animal study data to find out what dose of a drug can tolerate the body and what are its acute side effects. As a Phase 1 trial continues, researchers find out research mechanism of action, the side effects accompanying with increase in dosage, and information about effectiveness. This is imperative to the design of Phase 2 studies. Almost $70 \%$ of drugs travel to the next phase.

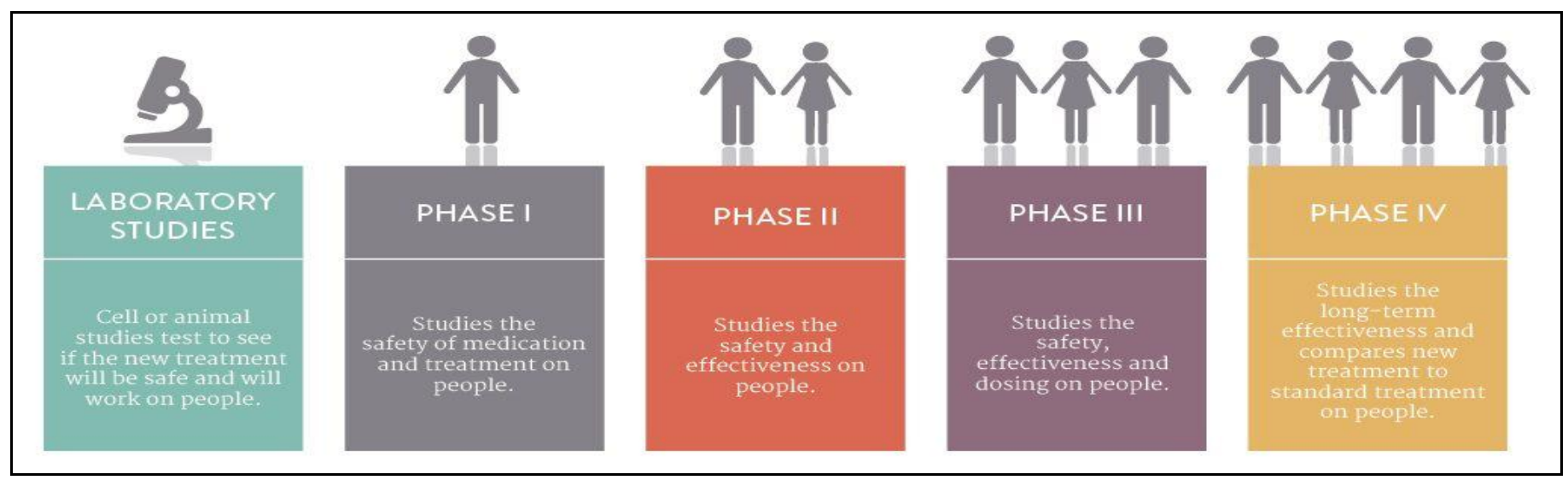

Figure 2: Phases of clinical trials 


\section{Phase 2: Efficacy and side effects}

Phase II trials are conducted on larger groups of patients (few hundreds) and are aimed to evaluate the efficacy of the drug and to endure the Phase I safety assessments. These trials aren'tsufficient to confirm whether the drug will be therapeutic. Phase 2 studies provide with additional safety data to the researchers. Researchers use these data to refine research questions, develop research methods, and design new Phase 3 research protocols. Around 33\% of drugs travel to the next phase.

Most prominently, Phase II clinical studies aid to found therapeutic doses for the large-scale Phase III studies.

\section{Phase 3: Efficacy and adverse drug reactions monitoring}

Researchers plan Phase 3 studies to prove whether a product deals anaction benefit to a specific peopleor not. Sometimes known as pivotal studies, these studies comprise 300 to 3,000 volunteers. Phase 3 studies deliver most of the safety data. Theprevious study might not able to detect less common side effects.Butphase 3 studies are conducted on large no. of volunteers and longer in duration, the results are more probable to detect long-term or uncommon side effects. Around $25-30 \%$ of drugs travel to the next phase of clinical research.

If a drug developer has data from its previous tests, preclinical and clinical trials that a drug is safe and effective for its intended use, then the industry can file an application to market the medicine. The FDA review team comprehensivelyinspects all submitted data on the drug and makes a conclusion to approve or not to approve it. ${ }^{[25]}$

\section{New Drug Application}

A New Drug Application (NDA) expresses the full story of a drug molecule. Its purpose is to verify that a drug is safe and effective for its proposed use in the people studied. A drug developer must include all about a drug starting from preclinical data to Phase 3 trial datain the NDA. Developers must include reports on all studies, data, and analysis. ${ }^{[26]}$ Beside with clinical trial outcomes, developers must include:

- Proposed labeling

- Safety updates

- Drug abuse information

- Patent information

\section{REFERENCES}

1. Shayne CG. Introduction: drug Discovery in the $21^{\text {st }}$ Century. Drug Discovery Handbook, Wiley Press, 2005; 1-10.

2. Smith GC, OíDonnel JT. The Process of New Drug Discovery and Development, Eds., 2nd edition, Informa Healthcare, New York 2006.

3. Moffat J, Vincent F, Lee J, Eder J, Prunotto M. Opportunities and challenges in phenotypic drug discovery: an industry perspective. Nature Reviews Drug Discovery, 2017; 16(8):531-543.

4. DiMasi JA, Hansen RW, Grabowski HG. The price of innovation: new estimates of drug development costs. Journal of Health Economics, 2003; 151-185.

5. Gashaw I, Ellinghaus P, Sommer A, Asadullah K. What makes a good drug target. Drug Discovery Today, 2012; 17:S24-S30.
- Institutional review board compliance information

- Directions for use

FDA Review

Once FDA obtains a complete NDA then FDA team of review may require about 6 to 10 months to take a pronouncement on whether to approve the NDA. If Once FDA obtains a incomplete NDA then FDA team of review refuse the NDA.

If FDAgoverns that a drug has been revealed to be safe and effective for its proposed use, it is then essential to work with the developerforupgrade prescribing information. This is denoted as "labeling." Labeling preciselydefines the basis for approval and directionhow to use the drug. Although, remaining issues required to be fixed before the drug to be approved for marketing. In other cases, FDA have need of additional studies. At this situation, the developer can choose whether to continue further developmentor not. If a developer distresses with an FDA decision, there are tools for official appeal. ${ }^{[27]}$

\section{Phase 4: Post-Market Drug Safety Monitoring}

Phase 4 trials are conductedwhen the drug or devicehas been approved by FDA.These trials are also recognized as postmarketing surveillance involving pharmacovigilance and continuing technical support after approval. There are numerous observational strategies and assessmentpatterns used in Phase 4trials to evaluate the efficacy, costeffectiveness, and safety of an involvement in real-world settings. Phase IV studies may be required by regulatory authorities (e.g. change in labelling, risk management/minimization action plan) or may be undertaken by the sponsoring company for competitive purposes or other reasons. Therefore, the true illustration of a drug's safety essentiallyrequires over the months and even years that mark up a drug'slifespan in the market. FDA reviews reports of complications with prescription and OTC drugs, and can decide to add precautions to the dosage or practice information, as well as other events for more serious adverse drug reactions. ${ }^{[28]}$

\section{ACKNOWLEDGEMENT}

We all express heartfelt gratitude to Dr. Nitin Hire, Principal of MVP's Institute of Pharmaceutical Sciences, Adgaon for their guidance and also for providing digital library.

6. Lindsay MA. Target discovery. Nature Reviews Drug Discovery, $2003 ; 2: 831-838$.

7. Terstappen G, Schlüpen, C, Raggiaschi R, Gaviraghi G. Target deconvolution strategies in drug discovery. Nature Reviews Drug Discovery, 2007; 6(11):891-903.

8. Peet NP. What constitutes target validation? Targets. 2003; 2:125127.

9. Imming P, Sinning C, Meyer A. Drugs, their targets and the nature and number of drug targets. Nature Reviews Drug Discovery, 2006; 5:821834.

10. Odilia Osakwe. Social Aspects of Drug Discovery, Development and Commercialization. Chapter 6 Preclinical In Vitro Studies: Development and Applicability. Elsevier. 2016. 
11. Croston G. The utility of target-based discovery. Expert Opinion on Drug Discovery, 2017; 12(5):427-429.

12. Henning SW, Beste G. Loss-of-function strategies in drug target validation. Current Drug Discovery Technology, 2002; 17-21.

13. John GH, Martyn NB, Bristol-Myers S. High throughput screening for lead discovery. Burger's Medicinal Chemistry and Drug Discovery, $6^{\text {th }}$ edition, Drug Discovery and Drug Development, Wiley Press, 2002; 2:37-70.

14. Patidar AK, Selvam G, Jeyakandan M, Mobiya AK, Bagherwal A, Sanadya G, Mehta R. Lead Discovery and lead optimization: A useful strategy in molecular modification of lead compound in analog design. International journal of drug design and discovery. 2011; 2(2):458463.

15. Huber W. A new strategy for improved secondary screening and lead optimization using high-resolution SPR characterization of compoundtarget interactions. J Mol. Recogn. 2005; 18:273-281.

16. Lofas S. Optimizing the hit-to-lead process using SPR analysis. Assay of Drug Development Technologies, 2004; 2:407-416.

17. Barile FA. Pri. nciples of Toxicological Testing. CRC Press, USA, 2008.

18. Friedman LM, Furberg CD, Demets DL. Fundamentals of clinical trials. 4th ed. New York: Springer Science and Business Media LLC; 2010

19. Faqi AS. A comprehensive guide to toxicology in preclinical drug development. Waltham, MA: Elsevier; 2013.
20. Vogel HG. Drug Discovery and Evaluation $2^{\text {nd }}$ edition. Springer, USA, 2002.

21. Karara AH, Edeki T, McLeod J, et al. PhRMA survey on the conduct of first-in-human clinical trials under exploratory investigational new drug applications. Journal of ClinicalPharmacology, 2010; 50:380391.

22. Fitzpatrick S. The clinical trial protocol. Buckinghamshire: Institute of Clinical Research; 2005.

23. Kinders, Robert, et al. Phase 0 Clinical Trials in Cancer Drug Development: From FDA.

24. DiMasi J. Risks in New Drug Development: Approval success Rates for Investigational Drugs. Clinical Pharmacology \& Therapeutics, 2001; 297-307.

25. Friedhoff L. New Drugs: An Insider's Guide to the FDA's New Drug Approval Process for Scientists, Investors and Patients. New York, NY: PSPG Publishing; 2009.

26. FDA (2003). New Drug Approval Reports. http://www.fda.gov/cder/rdmt/default.htm.

27. FDA, The FDA and the Drug Development Process: How the FDA insures that drugs are safe and effective, FDA Fact sheet, 2002.

28. Adams CP, and Brantner VV. New Drug Development: Estimating entry from human clinical trials. Bureau of Economics Federal Trade Commission. 2003. 